\title{
Bipartite promoters and RNA editing of paramyxoviruses and filoviruses
}

\author{
PHILIPPE LE MERCIER ${ }^{1}$ and DANIEL KOLAKOFSKY ${ }^{2}$ \\ ${ }^{1}$ Swiss-Prot Group, Swiss Institute of Bioinformatics, CMU, 1211 Geneva, Switzerland \\ ${ }^{2}$ Department of Microbiology and Molecular Medicine, University of Geneva Medical School, 1211 Geneva, Switzerland
}

\begin{abstract}
A primary property of paramyxovirus bipartite promoters is to ensure that their RNA genomes are imprinted with a hexamer phase via their association with nucleoproteins, in part because this phase as well the editing sequence itself controls mRNA editing. The question then arises whether a similar mechanism operates for filoviruses that also contain bipartite promoters that are governed by the "rule of six," even though these genomes need not, and given Ebola virus biology, cannot always be of hexamer genome length. This review suggests that this is possible and describes how it might operate, and that RNA editing may play a role in Ebola virus genome interconversion that helps the virus adapt to different host environments.
\end{abstract}

Keywords: Ebola virus; RNA editing; bipartite promoters; genome interconversion; rule of six

\section{INTRODUCTION}

Negative strand RNA viruses (NSV) are classified into those with segmented (sNSV) and nonsegmented genomes (nsNSV). The latter contain 5-10 genes in tandem on a genome roughly $10-20 \mathrm{~kb}$ in length. They include the families Bornaviridae, Rhabdoviridae (rabies virus and VSV), Pneumoviridae (respiratory syncytial virus [RSV]), Filoviridae (Ebola and Marburg virus), and the extensive Paramyxoviridae with several genera, including Respirovirus (Sendai virus [SeV] and parainfluenza virus type 3 [PIV3]), Morbillivirus (measles virus), and Rubulavirus (mumps virus and PIV2 and 5), all within the order Mononegavirales. Except for bornaviruses that replicate in the nucleus and have access to cellular RNA splicing, the others are restricted to the cytoplasm and use other mechanisms to express overlapping open reading frames, such as alternate ribosomal initiation codons and ribosomal frameshifting. Two nsNSV families, paramyxoviruses and filoviruses, uniquely use yet another mechanism to control gene expression, referred to as cotranscriptional RNA editing, or transcriptional frameshifting. In this process, viral RdRp is proposed to pause at a unique site during transcription in response to a cis-acting sequence, and to add a defined number of purine nucleotides to the transcript (guanosines for paramyxoviruses and adenosines for Ebola virus [EBOV]) before continuing elongation in a strictly template-dependent manner (Vidal et al. 1990).

Corresponding author: daniel.kolakofsky@unige.ch

Article is online at http://www.rnajournal.org/cgi/doi/10.1261/rna. 068825.118.

\section{PARAMYXOVIRUSES}

nsNSV genomes are encapsidated within a noncovalent chain of viral nucleoprotein $(\mathrm{N})$, forming a relatively regular helical structure: the nucleocapsid or N-RNA. The ssRNA genome is found within a positively charged groove of the protomer chain, between the two lobes of $N$ (Ruigrok et al. 2011). This protomer chain is held together primarily by amino- and carboxy-terminal arms of $\mathrm{N}$, which bridge adjacent protomers. For filoviruses, the intercalation of surface $\alpha$-helices of adjacent protomers is important (Wan et al. 2017; Sugita et al. 2018). Each protomer of paramyxovirus N-RNAs is associated with precisely $6 \mathrm{nt}$, and overall genome length must be precisely a multiple of $6 \mathrm{nt}$ (Egelman et al. 1989; Calain and Roux 1993; Kolakofsky et al. 1998). Only genomes whose lengths are multiples of $6 \mathrm{nt}$ are found naturally or are replicated efficiently in transfected cell systems. When mini-genomes whose lengths were not multiples of six were examined, insertions or deletions occurred during antigenome synthesis which strikingly restored overall hexamer length (Hausmann et al. 1996; Skiadopoulos et al. 2003).

Paramyxovirus genome replication is governed by this "rule of six," which acts in part during promoter recognition and the initiation of RNA synthesis from the genome $3^{\prime}$ end. Because hexamer length $(6 n+0)$ genomes have a

(c) 2019 le Mercier and Kolakofsky This article is distributed exclusively by the RNA Society for the first 12 months after the full-issue publication date (see http://rnajournal.cshlp.org/site/misc/terms.xhtml). After 12 months, it is available under a Creative Commons License (Attribution-NonCommercial 4.0 International), as described at http:// creativecommons.org/licenses/by-nc/4.0/. 
strong replicative advantage, this rule imposes a hexamer phase on the entire RNA genome. The structural basis for this rule was deduced from the cryo-EM reconstruction of measles virus (MeV) N-RNA and the crystal structure of PIV5 N-RNA rings (Alayyoubi et al. 2015; Gutsche et al. 2015). Of the 6 nt associated with each protomer in both cases, three contiguous bases are stacked and pointing toward the solvent (or "out"), and the other three bases are stacked and point toward the protein core (or "in"). The specific register of the bases for $\mathrm{MeV}$ is hexamer positions 5 and 6 from the $3^{\prime}$ end (hex 5 and 6 ) and hex 1 of the next $\mathrm{N}$ protein point out, and hex 2 , 3 , and 4 point in $\left(5,6,1^{\text {out }} 2,3,4^{\text {in }}\right.$ $5,6,1^{\text {out }} 2,3,4^{\text {in }}$ ) (Fig. 1A). Consistent with this register, whereas all adenosines and cytosines in VSV N-RNA are mostly reactive with dimethyl sulfate, only cytosines in SeV N-RNAs are modified, and their reactivity is strongly dependent on their hexamer phase; only those at hex5, 6, and 1 are modified (Iseni et al. 2002). For PIV5, the register is unknown, and may be simply positions $1,2,3^{\text {in }}$ $4,5,6^{\text {out }}$. This alternating arrangement of inward and outward facing triplets is imposed on the bound RNA by the $\mathrm{N}$ protein and presumably found throughout the N-RNA.

Because paramyxovirus genomes are precisely of hexamer length, each nucleotide within cis-acting sequences also has a precise position within the binding groove of each protomer. This is particularly important for genome $3^{\prime}$ end promoters that are bipartite in nature, being composed (at a minimum) of the first $12 \mathrm{nt}$ at the very genome $3^{\prime}$ end (promoter element 1

[PE1]). The second component (PE2) is found in the $5^{\prime}$-UTR of the first $(N)$ gene and the $3^{\prime}$-UTR of the last $(L)$ gene. PE2 is simply the presence of cytosine at hex 1 of protomers 14, 15, and 16 (from the genome $3^{\prime}$ end) for $\mathrm{SeV}$ and PIV3 (respiroviruses) and MeV, a morbillivirus (Tapparel et al. 1998; Walpita 2004). Rubulavirus PIV5 PE2 is also very simple, GC at hex 5 and hex6 of protomers 13, 14, and 15 (Murphy and Parks 1999). As the paramyxovirus N-RNA contains 13 protomers per helical turn, PE1 and PE2 are found on the same vertical face of the N-RNA helix, such that viral RNA-dependent RNA polymerase (RdRp) can simultaneously interact with both promoter elements (Fig. 1A). Tellingly, the displacement of the PE2 tripartite repeat from its conserved positions by even one hexamer position strongly reduces genome replication, whereas its displacement by six positions is tolerated (Pelet et al. 1996; Murphy et al. 1998).

Recent studies with PIV2, a close relative of PIV5, suggest why its RdRp initiates RNA synthesis so poorly when the hexamer phase of the PE2 tripartite repeat is 
displaced. PE1 of $\mathrm{N}^{\mathrm{wt}}$-RNA was found to contain a negative element, which when mutated (e.g., $\mathrm{N}^{\mathrm{O} 202 \mathrm{~A}}$ ) allows robust PE2-independent RNA synthesis that is now also largely independent of overall genome length, that is, a strict rule of six no longer applies (Matsumoto et al. 2017, 2018). PE2 in $\mathrm{N}^{\text {wt }}$-RNAs is apparently needed to neutralize the negative effects of $P E 1$, ensuring that the conserved hexamer alignment of the PE2 tripartite repeat is critical for the initiation of RNA synthesis. As the hexamer phase of the editing sequence as well as the sequence itself controls mRNA editing, this helps explain why paramyxovirus $6 n+0$ genome length, bipartite replication promoters, and cotranscriptional mRNA editing are linked (Iseni et al. 2002; Kolakofsky 2016). Hexamer phasing of this cis-acting sequence requires hexamer length genomes, as these N-RNAs are assembled from their very RNA $5^{\prime}$ end and terminate at the RNA $3^{\prime}$ end. Bipartite promoters ensure that genomes with nonhexamer length insertions or deletions replicate poorly because such changes automatically alter the hexamer phase of the promoter elements. The hexamer phase of the cis-acting editing sequence, 2-3000 nt removed from the genome $3^{\prime}$ end, is thus conserved by that of PE1 and PE2.

\section{FILOVIRUSES}

Ebola viruses (EBOV) cotranscriptionally edit their glycoprotein (GP) mRNA, to express both full-length GP (receptor-binding protein), and soluble GP (sGP, a modulator of host immunity [de la Vega et al. 2015] that is also cleaved to produce the $\delta$ peptide, a viroporin [He et al. 2017]). EBOV RNA editing sites are composed in part of a simple run of either seven (EBOV/7U) or eight template uridines (EBOV/8U), and both genomes that differ by $1 \mathrm{nt}$ in overall length are present during infection. For EBOV/U8, realignment of the nascent transcript $3^{\prime}$ end, both upstream and downstream on the genome template, can occur during RNA editing. EBOV RdRp can then skip as well as re-read a template uridine during RNA editing, resulting in nucleotide deletions as well as insertions (Figs. 2 and 3). In further contrast to paramyxoviruses, this site is frequently edited during antigenome synthesis. During EBOV/7U infection, $26 \%$ of the mRNAs and $16 \%$ of the antigenomes contained eight adenosines at $24 \mathrm{~h}$ postinfection (Shabman et al. 2014). EBOV/8U outgrows and eventually replaces EBOV/7U in cell culture, whereas EBOV/8U infection of guinea pigs leads to the rapid predominance by EBOV/7U. 7U/8U genome interconversion is suggested to play a role in EBOV host adaptation, linked to the relative expression of GP and sGP (Volchkova et al. 2011, 2015a).

Remarkably, filovirus promoters are also bipartite in nature, in contrast to those of other nsNSV families, such as Rhabdoviridae (VSV) or Pneumoviridae (RSV) that do not edit their transcripts. Similar to paramyxoviruses, filovirus promoters consist of a first element located within the leader region, and a second downstream element in the $5^{\prime}$-UTR of the first (N) gene, separated by a spacer region. Again, while proper spacing of the two promoter elements is a prerequisite for replication, the sequence of the spacer region is not important. Experimentally, replication was observed when six or a multiple of $6 \mathrm{nt}$ were deleted or inserted in the spacer region; all other changes in spacer length abolished replication. EBOV PE2 is also composed of contiguous hexamer repeats (five to eight $3^{\prime} \mathrm{NNNU}^{4} \mathrm{NN}$ for $E B O V / 7 U$ ) that also begin at the 13th or 14th protomers from the genome $3^{\prime}$ end for the five EBOV strains or serotypes, but three repeats are sufficient for replication (Weik et al. 2005; Enterlein et al. 2009). EBOV bipartite promoters are thus organized and appear to operate similarly to those of paramyxoviruses except for the "elephant in the room," that their genomes need not, nor given EBOV biology, cannot always be precisely a multiple of $6 \mathrm{nt}$ long.

A primary property of paramyxovirus bipartite promoters is to ensure that their genomes are imprinted with a hexamer phase via their association with $\mathrm{N}$. When an ectopic editing site is added to the SeV L gene $3^{\prime}$-UTR, editing here switches from adding one guanosine at high frequency (like 


$$
\begin{aligned}
& \text { EBOV/7U } \\
& \text { 3' ACC CUUUGA UUUUUUU } \\
& \text { 5' OGGGGAAACU AAAAAA A } \\
& \text { realign upstream } \\
& \text { 3' ACC CUUUGA UUUUUUU UGGAGU GAUC } \\
& \text { 5' GGGAACUA AAAAAA }
\end{aligned}
$$

resume strictly-templated synthesis

3' ACC CUUUGA UUUUUU UGGAGU GAUC

$$
\begin{aligned}
& { }_{5}, G G G A A A C U A A A A A A ~ A ~ \\
& \text { EBOV/8U }
\end{aligned}
$$

\section{UUUGAU UUUUUU UGGAGU GAUC GGGAAACUA
EBOV/7U}

FIGURE 3. Interconversion of EBOV 7U/8U genomes. Models of the RNAs in and around RdRp's synthesis chamber at the editing pause site, the seventh uridine from the start of the $U$ run (highlighted in black). The nascent product strand is shown below, $5^{\prime}-3^{\prime}$, and the genome template strand $\left(3^{\prime}-5^{\prime}\right)$ is on top; the separations in the sequences indicate hexamer phase. The seventh $U$ (in bold black) is proposed as the site where RdRp pauses, forming a 7 bp RNA:RNA hybrid. Hybrid realignments that lead to adenosine insertions or deletions in the transcript can occur as indicated. See text for detail.

at the SeV P gene site; $\left.3^{\prime} U_{6} C_{3}\right)$, to adding one to six $G s$ at roughly equal frequency (like at the PIV3 $P$ gene site; $3^{\prime}$ $\mathrm{U}_{6} \mathrm{C}_{4}$ ), simply by altering its hexamer phase (Iseni et al. 2002). Cotranscriptional RNA editing requires RdRp to pause at the template pause site to allow the nascent transcript 3 ' end to realign with the template "slippery" sequence (Vidal et al. 1990). Hexamer phase is proposed to affect this editing by altering the pace at which the template pause site arrives at RdRp's active site, and how long it spends there before continuing strictly templated elongation downstream. The hexamer phase can act here during the separation of each $\mathrm{N}$ from its $6 \mathrm{nt}$ as the template RNA enters RdRp's narrow template entry channel on its way to the synthesis chamber in RdRp's core (Fig. 4; Reguera et al. 2016). The transiently RNA-free $N$ chain must follow template movement through RdRp by moving in synchrony across RdRp's surface, such that it is repositioned to rebind its cognate hexa-nucleotide as the template exits RdRp. Possible interaction of the RNAfree $\mathrm{N}$ chain with RdRp's surface during these coordinated translocations would also take place in a hexamer phase-dependent manner.

Obligatory $6 n+0$ genome length has obviously not been selected by Figure 3.
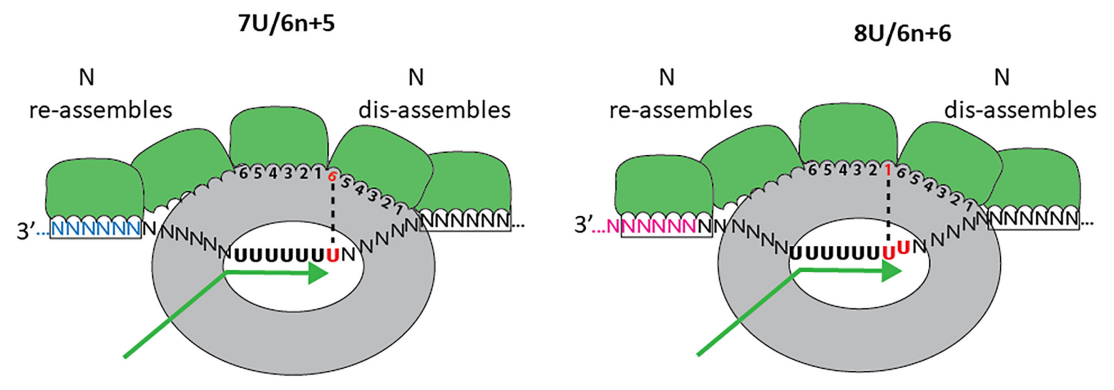

FIGURE 4. Possible ways in which hexamer phase can affect EBOV RNA editing. Cartoon of $U 7 / 6 n+5$ and U8/6n + 0 RNA synthesis elongation complexes when the active site of RdRp (internal oval) is paused at the proposed editing site; the seventh $U$ of the $U$ run, in red (vertical dashed lines). The eighth $U$ of $8 U / 6 n+0$ is also highlighted. The nascent transcript is shown below the template (bent arrow in green). During RNA synthesis, as RdRp moves (left to right) down the template, $\mathrm{N}$ protomers are continuously being separated from their associated nucleotides as the template RNA enters the template entry channel (on right side), and Ns reassociate with their cognate hexa-nucleotides as the template exits RdRp via its product exit channel (left side). These events must be highly coordinated, and these N:RNA interactions can affect the pace at which RdRp's active site arrives at, and remains at the pause site, before continuing strictly templated RNA synthesis. The template RNAs exiting RdRp are colored differently in U7/6n+ 5 and $U 8 / 6 n+0$ as they would be in different hexamer phases. The protomers of the RNA-free N chain are depicted crossing RdRp's top surface from right to left, and their hexamer positions are indicated. Possible protomer interactions with this surface will occur in a hexamer phase-dependent manner as schematized, and could affect how the nascent transcript $3^{\prime}$ end realigns with the template slippery sequence during the pause, as depicted in 
TABLE 1. Hexamer positions of EBOV cis-acting sequences

\begin{tabular}{|c|c|c|c|c|c|c|c|c|c|c|}
\hline & \multirow[b]{2}{*}{ Zaire } & \multirow[b]{2}{*}{ hex\# } & \multirow[b]{2}{*}{ Sudan } & \multirow[b]{2}{*}{ hex\# } & \multicolumn{2}{|c|}{ Ebolavirus } & \multicolumn{2}{|c|}{240 sequences } & \multirow[b]{2}{*}{ Tai Forest } & \multirow[b]{2}{*}{ hex\# } \\
\hline & & & & & Reston & hex\# & Bundibugy & hex\# & & \\
\hline $\mathrm{N}$ start & 56 & 4 & 56 & 4 & 56 & 3 & 56 & 4 & 56 & 4 \\
\hline VP35 start & 3032 & 4 & 3013 & 5 & 3003 & 2 & 3020 & 4 & 3026 & 4 \\
\hline VP40 start & 4390 & 2 & 4365 & 3 & 4396 & 1 & 4372 & 2 & 4378 & 2 \\
\hline GP start & 5900 & 4 & 5883 & 3 & 5901 & 2 & 5882 & 4 & 5888 & 4 \\
\hline GP editing site & 6918 & 6 & 6877 & 5 & 6924 & 5 & 6900 & 6 & 6906 & 6 \\
\hline GP Zaire second editing & 6378 & 6 & NA & & NA & & NA & & NA & \\
\hline Vp30 start & 8288 & 4 & 8224 & 2 & 8246 & 3 & 8269 & 5 & 8276 & 4 \\
\hline Vp24 start & 9885 & 3 & 9826 & 2 & 9832 & 1 & 9869 & 1 & 9873 & 3 \\
\hline L start & 11,501 & 1 & 11,457 & 3 & 11,471 & 6 & 11,487 & 3 & 11,486 & 4 \\
\hline Genome length $(6 n+X)$ & 18,959 & 5 & 18,875 & 5 & 18,892 & 4 & 18,941 & 5 & 18,935 & 5 \\
\hline
\end{tabular}

Nucleotide positions of EBOV transcription start and editing sites on the various negative-stranded $7 \mathrm{U}$ genomes (lefthand columns) are numbered from the genome $3^{\prime}$ ends. However, hexamer positions of these sites on the $7 \mathrm{U}$ genomes (hex\#, righthand columns) are numbered from their 5 -ends, where encapsidation is primed. Nearly complete genomes of 240 EBOV were aligned using MAFFT (PMID 28968734). Genomes missing a few nucleotides, either 3' or 5', were completed using consensus leader and trailer sequences. The alignment was used to identify the motifs of mRNA start and editing sites, and to record their positions relative to the genome $5^{\prime}$ end. The second GP editing site is only reported for Zaire (PMID 25370495). The results were similar in the five taxonomic groups: Zaire $(n=209)$, Sudan $(n=14)$, Reston $(n=8)$, Bundibugyo $(n=7)$, and Tai Forest viruses $(n=2)$. The bottom line displays overall genome lengths, both as total nucleotides and as $6 n+X$. The colors highlight the hexamer positions of the various cis-acting sequences and genome lengths. The darker color highlights their conservation as described in the text.

hex5 (counting from the genome $5^{\prime}$ end and the $3^{\prime} U$ of the $\mathrm{U}$ run).

2. Even though the $\mathrm{N}$ gene start sites are roughly 18,000 nt removed from their genome $5^{\prime}$ ends, four of these sites start at hex4 and one at hex3.

3. Even though their overall genome lengths are not precisely the same nor divisible by six, their overall lengths do not appear to be randomly distributed; four are $6 n+$ 5 and one at $6 n+4$ for EBOV/7U.

Compared to that of paramyxoviruses, EBOV RNA editing is more complex; deletions as well as insertions may occur during both mRNA and antigenome synthesis, possibly with both $7 \mathrm{U}$ and $8 \mathrm{U}$ genomes. It seems unlikely that all this takes place in a purely stochastic fashion, where only the stability of the template/nascent strand hybrid plays a role (Hausmann et al. 1999). A model for these events is shown in Figure 3, where the critical pause site for the $7 \mathrm{U}$ slippery sequence is the last $U$ for EBOV $7 U / 6 n+5$, allowing for a 7 bp RNA:RNA hybrid. This arrangement precludes nucleotide deletions, as the transcript $3^{\prime}$ end cannot realign downstream, according to the rule that only A:U, G:C, and G:U pairs in the hybrid are permitted (Vidal et al. 1990). Whether one or more adenosines (or none) are added during this transcript modification may simply depend on the time RdRp spends at the pause site. However, for EBOV U8/6n+0, the seventh $U$ is moved upstream by one hexamer position and the transcript $3^{\prime}$ end can now also realign downstream to delete $1 \mathrm{nt}$ (but can only do so once), converting U8 to U7. Notably, this transcript $3^{\prime}$ end can also realign upstream, converting $\cup 8$ to U9. Nevertheless, this latter realignment must occur infrequently, as U9 genomes are relatively rare (at $24 \mathrm{~h}$ post-7U infection, there are 20 times more mRNAs and antigenomes with eight adenosines than those that contain nine [Shabman et al. 2014]). This rarity is presumably for good reason; this editing site can also serve as a cryptic transcription termination/polyadenylation site (Volchkova et al. 2015b). Transcript realignment thus appears to operate differently in $\mathrm{U} 7$ and $\mathrm{U} 8$ genomes, and this editing site may be operating as a molecular switch, helping the virus adapt to different host environments.

The notion that hexamer phase might affect EBOV RNA editing is appealing, but how can such a phase be maintained without $6 n+0$ genome length? During nsNSV genome replication, the nascent replicate RNA is thought to be assembled with $\mathrm{N}$ from its $5^{\prime}$ end as the transcript exits RdRp's product exit channel. Each EBOV N binds $6 \mathrm{nt}$ at a time in tandem with its binding to the previously assembled protomers, forming a nascent N-RNA. Genome hexamer phase is then determined from the replicate RNA's $5^{\prime}$ end (Fig. 2). When EBOV genomes are less than $6 n+0$ in length, the $3^{\prime}$-terminal $\mathrm{N}$ will be associated with less than $6 \mathrm{nt}$, and the putative hexamer phases of cis-acting sequences will vary accordingly, for example, $3^{\prime} N_{N N U^{4} N N}$ versus $3^{\prime} N N^{3} N N N$ for PE2 for the examples shown in Figure 2. If EBOV promoter sequences in resting nucleocapsids are recognized similarly to those of paramyxoviruses, the strictly conserved uridine of the penta to octa-hexamer repeats should be found within the outward-facing triplet, and which cannot be in the same 
precise hexamer position for $7 U$ and $8 U$ viruses. The specific register on the bases of EBOV N-RNAs is not known, but as $\mathrm{E} 309$ of $\mathrm{N}_{\mathrm{n}-1}$ contacts the ribose of the first ( $\left.3^{\prime}\right)$ nucleotide of $N_{n}$ (Sugita et al. 2018), a register of $6,1,2^{\text {in }} 3,4.5^{\text {out }}$, a variation of that proposed for $\mathrm{MeV}$, is consistent with this structural arrangement. In this case, the strictly conserved uridine of the hexamer repeats would be at hex3 or hex4 for EBOV $7 U / 6 n+5$ and $8 U / 6 n+0$, respectively, and available for direct interaction with RdRp. The precise nucleotide distance between EBOV PE1 (e.g., the 3' $18 \mathrm{nt}$ of the template) and the obligatory triple hexamer repeat of PE2 in this model will be the same in U7 and U8 genomes. Their spatial orientations will still be determined by the helical structure of the N-RNA (Fig. 1B). Given the number of interactions between RdRp and the N-RNA presumably needed for RNA synthesis to initiate, a difference of only $1 \mathrm{nt}$ in PE1/PE2 spacing might be sensed by RdRp that requires exactly $6 n$ spacing for initiation. This would generate a hexamer phase independent of overall genome length, and provide an explanation for how EBOV genomes are governed by the rule of six even when they are not of hexamer length. Possible experiments to examine this-the rule of six model of filovirus replication -would include mini-genomes that have a $7 \mathrm{U}$ or $8 \mathrm{U}$ editing sequence and whose predicted pause sites are in various hexamer positions. These editing sites would precede a reporter gene whose open reading frame relative to that of the editing site can be varied, and scoring for both relative reporter gene expression and the number of adenosines at the antigenome complement of the editing site.

\section{ACKNOWLEDGMENTS}

We are grateful to Machiko Nishio, Wakayama, Japan and Laurent Roux, Geneva for their interest and suggestions.

\section{REFERENCES}

Alayyoubi M, Leser GP, Kors CA, Lamb RA. 2015. Structure of the paramyxovirus parainfluenza virus 5 nucleoprotein-RNA complex. Proc Natl Acad Sci 112: E1792-E1799. doi:10.1073/pnas. 1503941112

Calain P, Roux L. 1993. The rule of six, a basic feature for efficient replication of Sendai virus defective interfering RNA. J Virol 67: 48224830.

de La Vega MA, Wong G, Kobinger GP, Qiu X. 2015. The multiple roles of sGP in Ebola pathogenesis. Viral Immunol 28: 3-9. doi:10.1089/vim.2014.0068

Egelman EH, Wu SS, Amrein M, Portner A, Murti G. 1989. The Sendai virus nucleocapsid exists in at least four different helical states. $J$ Virol 63: 2233-2243.

Enterlein S, Schmidt KM, Schümann M, Conrad D, Krähling V, Olejnik J, Mühlberger E. 2009. The Marburg virus 3' noncoding region structurally and functionally differs from that of Ebola virus. $J$ Virol 83: 4508-4519. doi:10.1128/JVI.02429-08

Gutsche I, Desfosses A, Effantin G, Ling WL, Haupt M, Ruigrok RW, Sachse C, Schoehn G. 2015. Structural virology. Near-atomic
cryo-EM structure of the helical measles virus nucleocapsid. Science 348: 704-707. doi:10.1126/science.aaa5137

Hausmann S, Jacques JP, Kolakofsky D. 1996. Paramyxovirus RNA editing and the requirement for hexamer genome length. RNA 2: 1033-1045.

Hausmann S, Garcin D, Delenda C, Kolakofsky D. 1999. The versatility of paramyxovirus RNA polymerase stuttering. J Virol 73: 55685576.

He J, Melnik LI, Komin A, Wiedman G, Fuselier T, Morris CF, Starr CG, Searson PC, Gallaher WR, Hristova K, et al. 2017. Ebola virus $\delta$ peptide is a viroporin. J Virol 24: JVI.00438-17. doi:10.1128/ JVI.00438-17

Iseni F, Baudin F, Garcin D, Marq JB, Ruigrok RW, Kolakofsky D. 2002. Chemical modification of nucleotide bases and mRNA editing depend on hexamer or nucleoprotein phase in Sendai virus nucleocapsids. RNA 8: 1056-1067. doi:10.1017/S1355838202029977

Kolakofsky D. 2016. Paramyxovirus RNA synthesis, mRNA editing, and genome hexamer phase: a review. Virology 498: 94-98. doi: 10.1016/j.virol.2016.08.018

Kolakofsky D, Pelet T, Garcin D, Hausmann S, Curran J, Roux L. 1998. Paramyxovirus RNA synthesis and the requirement for hexamer genome length: the rule of six revisited. J Virol 72: 891-899.

Matsumoto Y, Ohta K, Kolakofsky D, Nishio M. 2017. A point mutation in the RNA-binding domain of human parainfluenza virus type 2 nucleoprotein elicits abnormally enhanced polymerase activity. $J$ Virol 91: e02203-16. doi:10.1128/JVI.02203-16

Matsumoto Y, Ohta K, Kolakofsky D, Nishio M. 2018. The control of paramyxovirus genome hexamer length and mRNA editing. RNA 24: 461-467. doi:10.1261/rna.065243.117

Murphy SK, Parks GD. 1999. RNA replication for the paramyxovirus simian virus 5 requires an internal repeated (CGNNNN) sequence motif. J Virol 73: 805-809.

Murphy SK, Ito Y, Parks GD. 1998. A functional antigenomic promoter for the paramyxovirus simian virus 5 requires proper spacing between an essential internal segment and the $3^{\prime}$ terminus. $J$ Virol 72: 10-19.

Pelet T, Delenda C, Gubbay O, Garcin D, Kolakofsky D. 1996. Partial characterization of a Sendai virus replication promoter and the rule of six. Virology 224: 405-414. doi:10.1006/viro.1996.0547

Reguera J, Gerlach P, Cusack S. 2016. Towards a structural understanding of RNA synthesis by negative strand RNA viral polymerases. Curr Opin Struct Biol 36: 75-84. doi:10.1016/ j.sbi.2016.01.002

Ruigrok RW, Crépin T, Kolakofsky D. 2011. Nucleoproteins and nucleocapsids of negative-strand RNA viruses. Curr Opin Microbiol 14: 504-510. doi:10.1016/j.mib.2011.07.011

Shabman RS, Jabado OJ, Mire CE, Stockwell TB, Edwards M, Mahajan M, Geisbert TW, Basler CF. 2014. Deep sequencing identifies noncanonical editing of Ebola and Marburg virus RNAs in infected cells. MBio 5: e02011. doi:10.1128/mBio.02011-14

Skiadopoulos MH, Vogel L, Riggs JM, Surman SR, Collins PL, Murphy BR. 2003. The genome length of human parainfluenza virus type 2 follows the rule of six, and recombinant viruses recovered from non-polyhexameric-length antigenomic cDNAs contain a biased distribution of correcting mutations. J Virol 77: 270-279. doi:10.1128/JVI.77.1.270-279.2003

Sugita Y, Matsunami H, Kawaoka Y, Noda T, Wolf M. 2018. CryoEM structure of the Ebola virus nucleoprotein-RNA complex at $3.6 \AA ̊$ resolution. Nature 563: 137-140. doi:10.1038/s41586-0180630-0

Tapparel C, Maurice D, Roux L. 1998. The activity of Sendai virus genomic and antigenomic promoters requires a second element past the leader template regions: a motif $(\mathrm{GNNNNN})_{3}$ is essential for replication. J Virol 72: 3117-3128. 
Vidal S, Curran J, Kolakofsky D. 1990. A stuttering model for paramyxovirus P mRNA editing. EMBO J 9: 2017-2022. doi:10.1002/ j.1460-2075.1990.tb08330.x

Volchkova VA, Dolnik O, Martinez MJ, Reynard O, Volchkov VE. 2011. Genomic RNA editing and its impact on Ebola virus adaptation during serial passages in cell culture and infection of guinea pigs. J Infect Dis 204: S941-S946. doi:10.1093/infdis/ jir321

Volchkova VA, Dolnik O, Martinez MJ, Reynard O, Volchkov VE. 2015a. RNA editing of the GP gene of Ebola virus is an important pathogenicity factor. J Infect Dis 212: S226-S233. doi:10.1093/ infdis/jiv309
Volchkova VA, Vorac J, Repiquet-Paire L, Lawrence P, Volchkov VE. 2015b. Ebola virus GP gene polyadenylation versus RNA editing. $J$ Infect Dis 212: S191-S198. doi:10.1093/infdis/jiv150

Walpita P. 2004. An internal element of the measles virus antigenome promoter modulates replication efficiency. Virus Res 100: 199211. doi:10.1016/j.virusres.2003.12.025

Wan W, Kolesnikova L, Clarke M, Koehler A, Noda T, Becker S, Briggs JAG. 2017. Structure and assembly of the Ebola virus nucleocapsid. Nature 551: 394-397. doi:10.1038/nature24490

Weik M, Enterlein S, Schlenz K, Mühlberger E. 2005. The Ebola virus genomic replication promoter is bipartite and follows the rule of six. $J$ Virol 79: 10660-10671. doi:10.1128/JVI.79.16.10660-10671.2005 

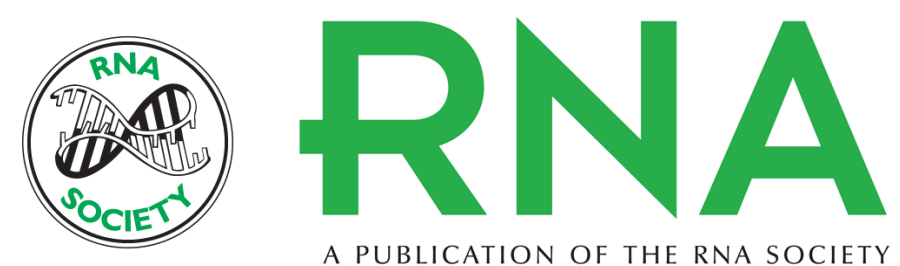

A PUBLICATION OF THE RNA SOCIETY

\section{Bipartite promoters and RNA editing of paramyxoviruses and filoviruses}

Philippe le Mercier and Daniel Kolakofsky

RNA 2019 25: 279-285 originally published online December 26, 2018

Access the most recent version at doi:10.1261/rna.068825.118

$\begin{array}{ll}\text { References } & \begin{array}{l}\text { This article cites } 30 \text { articles, } 17 \text { of which can be accessed free at: } \\ \text { http://rnajournal.cshlp.org/content/25/3/279.full.html\#ref-list-1 }\end{array}\end{array}$

Creative This article is distributed exclusively by the RNA Society for the first 12 months after the

Commons full-issue publication date (see http://rnajournal.cshlp.org/site/misc/terms.xhtml). After 12

License months, it is available under a Creative Commons License (Attribution-NonCommercial 4.0 International), as described at http://creativecommons.org/licenses/by-nc/4.0/.

Email Alerting Receive free email alerts when new articles cite this article - sign up in the box at the

Service top right corner of the article or click here.

To subscribe to RNA go to:

http://rnajournal.cshlp.org/subscriptions

(C) 2019 le Mercier and Kolakofsky; Published by Cold Spring Harbor Laboratory Press for the RNA Society 